13

\title{
Проводимость манганитных пленок под действием напряженности, вызванной деформацией подложки
}

\author{
(C) Г.А. Овсянников ${ }^{1}$, Т.А. Шайхулов ${ }^{1}$, В.А. Шахунов ${ }^{1}$, А.А. Климов ${ }^{1,3}$, В.Л. Преображенский ${ }^{2}$, \\ N. Tiercelin ${ }^{3}$, P. Pernod ${ }^{3}$ \\ ${ }^{1}$ Институт радиотехники и электроники им. В.А. Котельникова РАН, \\ Москва, Россия \\ ${ }^{2}$ Институт общей фризики РАН им. А.М. Прохорова, \\ Москва, Россия \\ ${ }^{3}$ University of Lille, CNRS, Centrale Lille, ISEN, Univ. Valenciennes, UMR 8520-IEMN, \\ F-59000 Lille, France \\ E-mail: gena@hitech.cplire.ru
}

Поступила в Редакцию 15 апреля 2019 г.

В окончательной редакции 22 апреля 2019 г.

Принята к публикации 24 апреля 2019 г.

\begin{abstract}
Представлены результаты исследования электронных транспортных свойств напряженных тонких эпитаксиальных пленок $\mathrm{La}_{0.7} \mathrm{Ba}_{0.3} \mathrm{MnO}_{3}$ (LBMO). Пленки толщиной 40-100 nm изготавливались с помощью лазерной абляции при температуре $T=700-800^{\circ} \mathrm{C}$ в атмосфере чистого кислорода $0.3-1 \mathrm{mBar}$. Для создания механического напряжения использовались подложки сегнетоэлектрического кристалла (011) $0.79 \mathrm{PbMg}_{1 / 3} \mathrm{Nb}_{2 / 3} \mathrm{O}_{3}-0.21 \mathrm{PbTiO}_{3}$ (PMN-PT) с температурой Кюри $150^{\circ} \mathrm{C}$ и высокими пьезоэлектрическими постоянными. Исследовалось влияние сегнетоэлектрической поляризации и пьезоэлектрического эффекта на электрические параметры LBMO пленок.
\end{abstract}

Ключевые слова: эпитаксиальные манганитные пленки, лазерная абляция, пьезоэлектрики.

DOI: $10.21883 /$ FTT.2019.09.48115.08N

\section{1. Введение}

Как было показано в ряде работ [1-6], причиной изменения магнитных параметров редкоземельных манганитных пленок перовскитов со структурой $R e_{1-x} A_{x} \mathrm{MnO}_{3}$ $(R e-$ редкоземельные элементы типа La или $\mathrm{Nd}$, a $A-$ щелочноземельные металлы типа $\mathrm{Sr}, \mathrm{Ca}, \mathrm{Ba})$ является механическое напряжение, вызванное рассогласованием параметров решетки пленки и подложки. Было установлено, что трехмерное сжатие кристаллической решетки увеличивает амплитуду вероятности перескока в модели двойного обмена, что ведет к увеличению температуры Кюри $\left(T_{c}\right)$, в то же время двухосные искажения ян-телеровского типа вызывают усиление локализации электронов и уменьшают $T_{c}$ [7-10]. Хотя в рамках модели двойного обмена магнитные и резистивные параметры тесно связаны, отсутствует ясность в механизме изменении резистивных свойств эпитаксиальных манганитных пленок при воздействии деформации. В тоже время управление резистивными состояниями эпитаксиальных манганитных пленок с помощью деформаций представляет интерес как одно из направлений развития стрейнтроники [11-13].

Манганитные пленки, для которых температура Кюри $T_{c}$ близка к комнатной, особенно привлекательны для практически применений. Ферромагнитный фазовый переход для однофазного кристалла $\mathrm{La}_{0.7} \mathrm{Ba}_{0.3} \mathrm{MnO}_{3}$ (LBMO) происходит при $T_{c}=345 \mathrm{~K}$ [14-17]. В монокристалле LBMO наблюдалась гигантская магнитострик- ция (до $4 \cdot 10^{-4}$ ) при температуре, равной температуре Кюри [18]. Ранее сообщалось, что магнитные и резистивные свойства эпитаксиальных LBMO пленок сильно изменяются при росте на разных подложках из-за влияния деформации решетки пленки, вызванной подложкой [19-21].

В данной работе представлены результаты экспериментального исследования электронных транспортных параметров эпитаксиальных манганитных пленок $\mathrm{La}_{0.7} \mathrm{Ba}_{0.3} \mathrm{MnO}_{3}$ (LBMO), выращенных на пьезоэлектрической подложке (011) $0.79 \mathrm{~Pb}\left(\mathrm{Mg}_{1 / 3} \mathrm{Nb}_{2 / 3}\right) \mathrm{O}_{3}-$ $0.21 \mathrm{PbTiO}_{3}$ (PMN-PT), которая обеспечивает деформацию пленки, управляемую электрическим полем.

\section{2. Экспериментальные образцы}

Эпитаксиальные $\quad \mathrm{La}_{0.7} \mathrm{Ba}_{0.3} \mathrm{MnO}_{3} \quad$ пленки толщиной 40-150 nm напылялись на подложки (011) $0.79 \mathrm{~Pb}\left(\mathrm{Mg}_{1 / 3} \mathrm{Nb}_{2 / 3}\right) \mathrm{O}_{3}-0.21 \mathrm{PbTiO}_{3} \quad$ размером $5 \times 5 \times 0.3 \mathrm{~mm} \mathrm{c}$ помощью лазерной абляции при температуре $600-800^{\circ} \mathrm{C}$ и давлении кислорода 0.2-0.5 mBar. Мишени изготавливались из смеси порошков $\mathrm{La}_{2} \mathrm{O}_{3}, \mathrm{BaCO}_{3}$ и $\mathrm{MnCO}_{3}$ по керамической технологии. Использовался стехиометрический состав смеси для проведения синтеза. В псевдокубическом представлении параметр решетки LBMO при стехиометрическом составе мишени по кислороду оказался равным $a_{L}=0.390 \mathrm{~nm}$. Примесь в мишени в виде окисла 


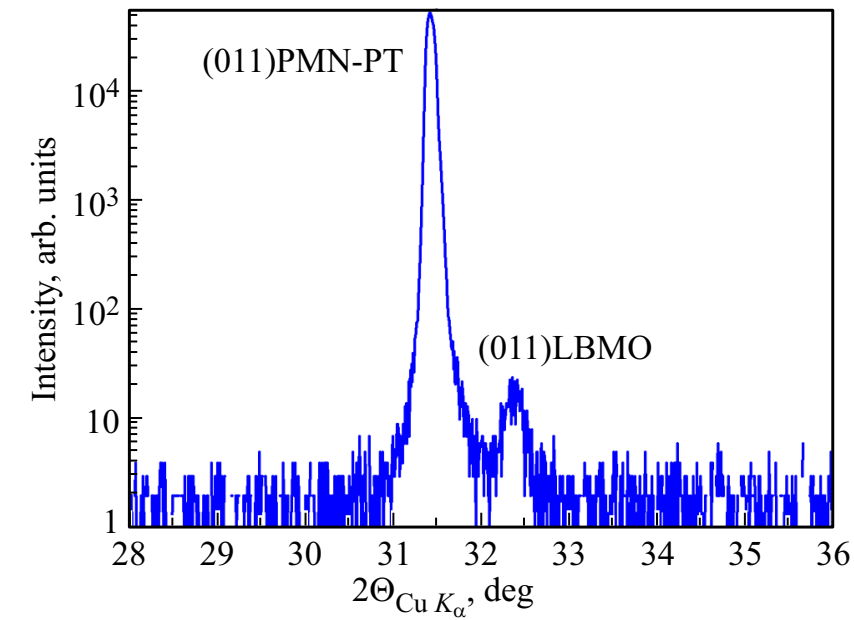

Рис. 1. $2 \Theta / \omega$ дифрактограмма пленки $\mathrm{La}_{0.7} \mathrm{Ba}_{0.3} \mathrm{MnO}_{3}$, напыленной на подложку (011) $\mathrm{PbMg}_{1 / 3} \mathrm{Nb}_{2 / 3} \mathrm{O} 3-\mathrm{PbTiO}_{3}$.

$\mathrm{BaMnO}_{3}$ составляла 2.5\% [20]. Сегнетоэлектрические кристаллы (PMN-PT) имеют поляризацию насыщения $P_{r}=30-35 \mathrm{C} / \mathrm{cm}^{2}$, низкое коэрцитивное поле и высокие пьезоэлектрические постоянные $d_{33}=1500 \mathrm{pC} / \mathrm{N}$, $d_{32}=-1883 \mathrm{pC} / \mathrm{N}$ и $d_{31}=610 \mathrm{pC} / \mathrm{N}[21,22]$.

Кристаллографические параметры пленок и подложек определялись с помощью 4-х кружного рентгеновского дифрактометра измерением рентгеновских дифрактограмм в режиме $2 \Theta / \omega$ и $\varphi$-сканирования, а также измерением кривых качания. Выращенные LBMO пленки были ориентированы как относительно нормали к плоскости подложки, так и относительно выделенного направления в плоскости подложки. Параметр межплоскостного расстояния в пленках LBMO вдоль нормали к плоскости подложки и постоянная решетки подложки определялись по $2 \Theta / \omega$ дифрактограммам рентгеновского сканирования. Представленный на рис. 1 участок $2 \Theta / \omega$ дифрактограммы пленки $\mathrm{La}_{0.7} \mathrm{Ba}_{0.3} \mathrm{MnO}_{3}$, напыленной на подложку (011) $\mathrm{PbMg}_{1 / 3} \mathrm{Nb}_{2 / 3} \mathrm{O}_{3}-\mathrm{PbTiO}_{3}$ показывает, что PMN-PT обладает структурой типа перовскита с близкой к кубу с постоянными решетки $a_{S}=0.403 \mathrm{~nm}$, a постоянная решетки пленки LBMO составляет $a_{L}=0.391 \mathrm{~nm}$. Рост пленки осуществляется по эпитаксиальному соотношению (011)LBMO//(011)PMN-PT, [100]LBMO//[100]PMN-PT. Значения возникающих в пленке напряжений в плоскости подложки в направлении [100] $\varepsilon_{100 \mid}=\left(a_{S}-a_{L}\right) / a_{L}$ в отсутствие электрического напряжения на подложке $\mathrm{PMN}-\mathrm{PT}$ составляет $3.1 \%$.

\section{3. Электрофизические параметры}

Сопротивление пленок изучалось четырехточечным методом, исключающим влияние контактного сопротивления. Контактные площадки изготавливались катодным напылением платины через металлическую маску. Передняя часть подложки, запыленная пленкой LBMO служила верхним электродом. На тыльную сторону подложки наносилась серебряная паста (см. рис. 2). Напряжение электрического смещения (около $100 \mathrm{~V}$ ) прикладывалось нормально к подложке. Сопротивление пленки измерялось при задании тока в плоскости подложки.

На температурных зависимостях сопротивления LBMO пленок в отсутствие электрического напряжения на подложке с уменьшением температуры ниже комнатной наблюдался пик сопротивления при $T=T_{M}$, которая является температурой перехода изолятор-металл (см. рис. 3). Кроме того, наблюдается анизотропия сопротивления по направлению протекания тока. Измерения проводились после преполяризации подложки

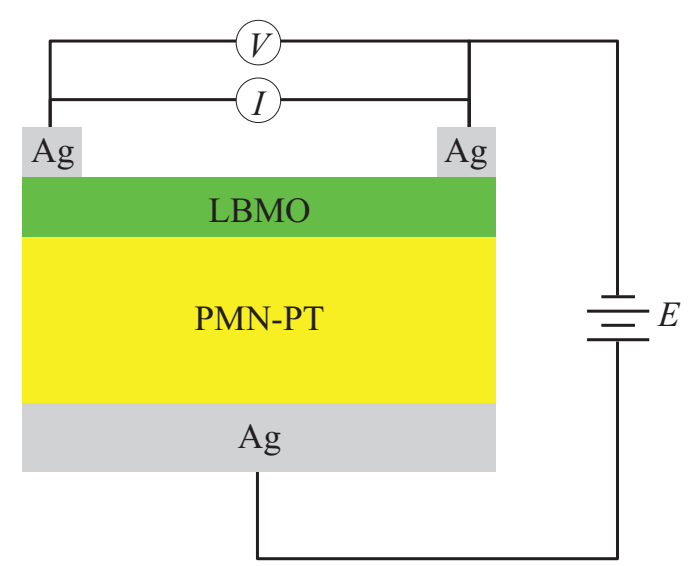

Рис. 2. Схема измерения зависимости сопротивления пленки LBMO от электрического поля, приложенного к подложке (011) PMN-PT. I - источник тока для измерения сопротивления, $V$ - измеритель напряжения для для измерения сопротивления, $E-$ источник высокого напряжения на подложки, вызывающий ее деформацию

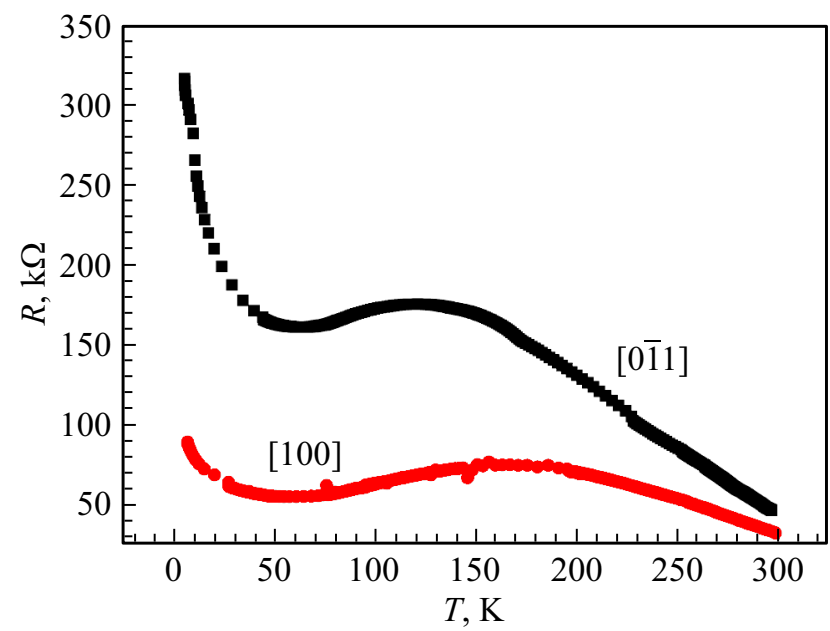

Рис. 3. Температурные зависимости сопротивления, измеренного при двух направлениях протекания тока в пленке $\mathrm{La}_{0.7} \mathrm{Ba}_{0.3} \mathrm{MnO}_{3}$, напыленной на подложку (011) $\mathrm{PbMg}_{1 / 3} \mathrm{Nb}_{2 / 3} \mathrm{O}_{3}-\mathrm{PbTiO}_{3}$. 
напряжением выше $100 \mathrm{~V}$, которая немного изменяет форму температурной зависимости сопротивления $R(T)$. Уменьшение сопротивления при $T \leq T_{M}$, скорее всего, вызвано увеличением площади проводящих ферромагнитных областей. При низких температурах $(T<100 \mathrm{~K})$ удельное сопротивление всех образцов увеличивается. Как было показано в [23], в кристаллах $\mathrm{La}_{1-x} \mathrm{Ba}_{x} \mathrm{MnO}_{3}$ с меньшим содержанием Ва $(x \leq 0.2)$ температура $T_{M}$ на несколько десятков градусов меньше, чем при оптимальном составе $(x=0.3)$, а при $T<T_{M}$ сопротивление LBMO пленок увеличивается. В пленках с оптимальным содержанием $\mathrm{Ba}(x=0.3)$ сопротивление при низких температурах определяется рассеянием носителей на примесях и дефектах и зависит от интенсивности электрон-электронного и электрон-магнонного взаимодействий [20]. В области высоких температур $T>T_{M}$ температурная зависимость сопротивления манганитных пленок определяется процессами активации [24].

Деформация подложки $\mathrm{PMN}-\mathrm{PT}$ измерялась с помощью тензодатчика, приклеенного к подложке PMN-PT [21], которые дают относительную деформацию подложки. Абсолютная деформация подложки не измерялась. Имеют смысл только относительные изменения. В образце наблюдается деформация растяжения подложки $(1000 \mathrm{ppm})$ в направлении [011] $\mathrm{PMN}-\mathrm{PT}$ при напряженности поля, равной коэрцитивной силе сегнетоэлектрической петли как при положительном $E_{c^{+}}=2 \mathrm{kV} / \mathrm{cm}$, так и при отрицательном $E_{c^{-}}=-2 \mathrm{kV} / \mathrm{cm}$ направлении поля. Деформация сжатия вдоль направления [100] PMN-PT существенно меньше (около $500 \mathrm{ppm}$ ) и наблюдается также при напряженности электрического поля, равной коэрцитивной силе, хотя видна асимметрия по знаку электрического поля. Наблюдаемые пики деформации подложки при напряженности поля вблизи коэрцитивной силы скорее всего вызваны вращением вектора сегнетоэлектрической поляризации $[25,26]$. Асимметрия наблюдаемых пиков относительно направления электрического поля вызваны типом измерения сопротивления пленки LBMO, вкладом растекания тока в поперечном к измеряемому направления. Следует обратить внимание, что в направлении [011] PMN-PT в отсутствие электрического поля отсутствует деформация подложки. Следовательно в отсутствие поля должны отсутствовать резистивные состояния в пленке LBMO.

Результаты измерений зависимости изменения сопротивления LBMO пленки от напряженности электрического поля для двух направлений протекания тока [0111] LBMO и [100] LBMO представлены на рис. $4, b$. Видно, что в целом изменение сопротивления LBMO пленки соответствует деформации подложки: имеется большое изменение сопротивления пленки, измеренного

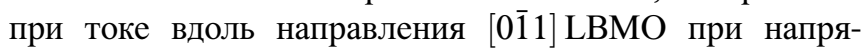
женности поля вблизи коэрцитивной силы. Наиболее сильное изменение сопротивления LBMO пленки на-

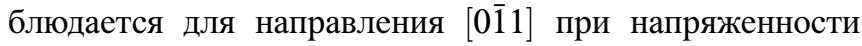
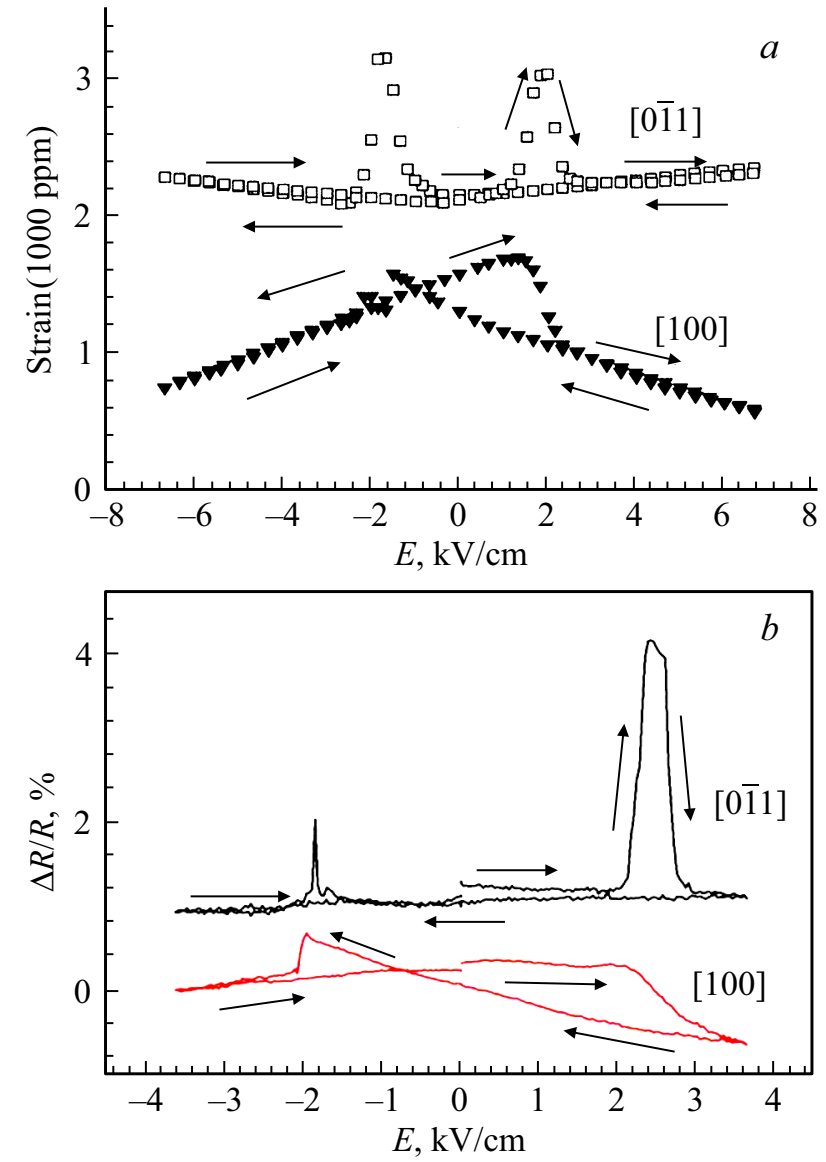

Рис. 4. $a-$ деформация подложки (011)PMN-PT, измеренная в направлениях [0̄ㅣ $\mathrm{PMN}-\mathrm{PT}$ и [100] PMN-PT, $b-$ зависимость сопротивления пленки $\mathrm{La}_{0.7} \mathrm{Ba}_{0.3} \mathrm{MnO}_{3}$ от напряженности приложенного электрического поля для двух направлений протекания тока. Кривые смещены по оси ординат для ясности. Важны относительные значения изменения как деформации, так и сопротивления имеют смысл. ( $E$ изменялось сначала в интервале от 0 до $4 \mathrm{kV} / \mathrm{cm}$, затем от $4 \mathrm{kV} / \mathrm{cm}$ до $-4 \mathrm{kV} / \mathrm{cm}$ и от $-4 \mathrm{kV} / \mathrm{cm}$ до $0 \mathrm{kV} / \mathrm{cm})$.

электрического поля, равного коэрцитивной силе сегнетоэлектрической петли. Результаты согласуются с представленными на рис. 4, $a$ зависимостями деформаций подложки в направлениях $[0 \overline{1} 1]$ и [001] от напряженности электрического поля. Максимальное значение изменения сопротивления, равное $3 \%$, сравнимо с величиной, наблюдавшейся в других исследованиях $[27,28]$.

Изменение сопротивления LBMO пленки может быть объяснено моделью двойного обмена [29], в рамках которой должна наблюдаться сильная зависимость сопротивления от напряженности пленки. Туннелирование между состяниями $\mathrm{Mn}^{3+}$ и $\mathrm{Mn}^{4+}$ зависит от расстояния между $\mathrm{Mn}$ и О. Это расстояние изменяется за счет растяжения LBMO пленки, которое увеличивает расстояние $\mathrm{Mn}-\mathrm{O}$ связей и уменьшает длину прыжка взаимодействия между состояниями $\mathrm{Mn}^{3+}$ и $\mathrm{Mn}^{4+}$, увеличивая сопротивление $[10,29]$. В работе [10] было показано, 


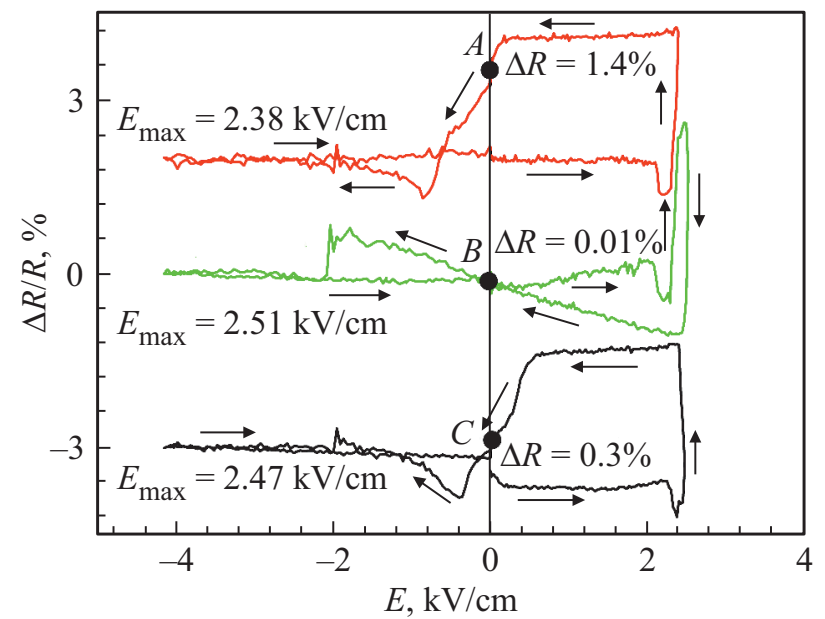

Рис. 5. Зависимость сопротивления пленки от напряженности электрического поля при асимметричном свипировании для трех значений максимального положительного электрического поля $E_{\max }=2.38,2.47$ и $2.51 \mathrm{kV} / \mathrm{cm}$. Измерения проводятся при протекании тока вдоль направления [01ㅣ LBMO. Кривые смещены по оси ординат для ясности. Относительные изменения как деформации, так и сопротивления имеют значения. $(E$ изменялось сначала в интервале от 0 до $4 \mathrm{kV} / \mathrm{cm}$, затем от $4 \mathrm{kV} / \mathrm{cm}$ до $-4 \mathrm{kV} / \mathrm{cm}$ и от $-4 \mathrm{kV} / \mathrm{cm}$ до $0 \mathrm{kV} / \mathrm{cm})$.

что в дополнение к механизму двойного обмена играет роль сильное электрон-фононное взаимодействие, возникающее из-за ян-теллеровского расщепления внешних орбиталей Mn.

При асимметричном свипировании электрического поля от $-4 \mathrm{kV} / \mathrm{cm}$ до одного из трех максимальных значений положительного электрического поля $E_{\max }=2.38$, 2.47 и $2.51 \mathrm{kV} / \mathrm{cm}$ наблюдаются несколько состояний с различным сопротивлением (А, В и С на рис. 5). При выключении электрического поля сопротивление пленки LBMO $(\Delta R / R)$ остается постоянным. Это состояние „сбрасывается“ при отрицательном значении электрического поля $(4 \mathrm{kV} / \mathrm{cm})$. Резистивное состояние с максимальным значением изменения сопротивления пленки $\Delta R / R=1.4 \%$ наблюдается при максимальном положительном значении напряженности электрического поля $E_{\max }$ немного меньше $E_{c^{+}}$. При больших значениях $E_{\max }$ величина сопротивления резистивного состояния либо уменьшается до долей процента, либо отсутствует. Аналогичная картина наблюдается при асимметричном свипировании электрического поля в отрицательную сторону. Величина $\Delta R / R$ при отрицательном свипировании существенно меньше из-за асимметрии зависимости сопротивления от поля (см. рис. 4,b). Такое поведение резистивных параметров LBMO пленки может быть объяснено сегнетоэлектрической переполяризации доменов [01ㅣ PMN-PT вокруг изоморфной фазовой границы [26-28]. При задании поля вдоль [100] аналогичные состояния существенно меньше по амплитуде и плохо воспроизводимы.
Таким образом, в LBMO пленке существует начальное состояние сопротивление LBMO пленки, обозначенное как „В“ на рис. 5, которое не зависит от напряжения, превышающего коэрцитивную силу как в положительном, так и в отрицательном направлении. При асимметричном свипировании кроме того существуют два других состояния (обозначены как „А“ и „С“ на рис. 5), которые имеют сопротивление отличное от „В“ состояния. Все три состояния достигаются изменением амплитуды свипирования электрического поля.

\section{4. Заключение}

В эпитаксиальных LBMO пленках, напыленных на подложку (011) PMN-PT, наблюдается анизотропия сопротивления, вызванная кристаллографической анизотропией выращенной пленки (110) LBMO. На температурных зависимостях сопротивления LBMO пленок в отсутствие напряженности подложки с уменьшением температуры ниже комнатной наблюдался пик сопротивления при $T=T_{M}$, типичный для манганитов. При низких температурах $(T<100 \mathrm{~K})$ сопротивление всех образцов увеличивается, что объясняется меньшим, чем в мишени, содержанием Ва. При симметричном свипировании напряженности электрического поля относительно нулевого значения в окрестности коэрцитивной силы наблюдается изменение сопротивления на уровне $3 \%$. При асимметричном свипировании на зависимости сопротивления пленки от поля наблюдаются устойчивые резистивные состояния, которые можно переключать изменяя амплитуду вариации электрического поля.

\section{Благодарности}

Авторы благодарны Н.В. Андрееву, К.И. Константиняну, А.М. Петржику, Е.А. Свиридовой, А.В. Шадрину за полезное обсуждение полученных результатов и помощь при проведении исследований.

\section{Финансирование работы}

Работа выполнена в рамках Государственного задания и частично поддержана грантами РФФИ 16-29-14022 и 19-07-00143 и Международной лабораторией LEMAC-LICS.

\section{Конфликт интересов}

Авторы заявляют, что у них нет конфликта интересов.

\section{Список литературы}

[1] A.-M. Haghiri-Cosnetand, J.P. Renard. J. Phys. D 36, R127 (2003).

[2] Zh. Huang, G.Y. Gao, Zh.Zh. Yin, X.X. Feng, Y.Zh. Chen, X.R. Zhao, J.R. Sun, W.B. Wu. J. Appl. Phys. 105,113919 (2009). 
[3] Г.А. Овсянников, А.М. Петржик, И.В. Борисенко, А.А. Климов, Ю.А. Игнатов, В.В. Демидов, С.А. Никитов. ЖЭТФ 135, 56 (2009).

[4] P.R. Sagdeo, N.P. Lalla, A.V. Narlika, D. Prabhakaran, A.T. Boothroyd. Phys. Rev. B 78, 174106 (2008).

[5] P. Orgiani, A. Guarino, C. Aruta, C. Adamo, A. Galdi, A.Yu. Petrov, R. Savo, L. Maritato. J. Appl. Phys. 101, 033904 (2007).

[6] P. Murugavel, T.W. Noh, J.G. Yoon. J. Appl. Phys. 95, 2536 (2004).

[7] Y. Lu, J. Klein, C. Hofener, B. Wiedenhorst, J.B. Philipp, F. Herbstritt, A. Marx, L. Alff, R. Gross. Phys. Rev. B 62, 15806 (2000).

[8] Y. Chen, B.G. Ueland, J.W. Lynn, G.L. Bychkov, S.N. Barilo, Y.M. Mukovskii. Phys. Rev. B 78, 212301(2008).

[9] F. Tsui, M.C. Smoak, T.K. Nath, C.B. Eom. Appl. Phys. Lett. 76, 2421 (2000).

[10] A.J. Millis, T. Darling, A. Migliori. J. Appl. Phys. 83, 1588 (1998).

[11] V. Iurchuk, B. Doudin, B. Kundys, D. Multistate. J. Phys.: Condens. Matter 26, 292202 (2014).

[12] Y. Lee, Z. Q.Liu, J.T. Heron, J.D. Clarkson, J. Hong, C. Ko, M.D. Biegalski, U. Aschauer, S.L. Hsu, M.E. Nowakowski, J. Wu, H. M. Christen, S. Salahuddin, J.B. Bokor, N.A. Spaldin, D.G. Schlom, R. Ramesh. Nature Commun. 6, 5959. (2015).

[13] Y. Yang, Z.L. Luo, M.M. Yang, H. Huang, H. Wang, J. Bao, G. Pan, C. Gao, Q. Hao, S. Wang, M. Jokubaitis, W. Zhang, G. Xiao, Y. Yao, Y. Liu, X.G. Li. Appl. Phys. Lett. 102, 33501 (2013).

[14] H.L. Ju, J. Gopalakrishnan, J.L. Peng, Qi Li, G.C. Xiong, T. Venkatesan, R.L. Greene. Phys. Rev. B 51, 6143 (1995).

[15] K. Rogacki, X. Xiong, P.W. Klamut, R. Dybzinski, J. Shaffer, J.D. Jorgensen, B. Dabrowski. Phys. Rev. B 58, 2716 (1998).

[16] H.L. Ju, Y.S. Nam, J.E. Lee, H.S. Shin. J. Magn. Magn. Mater. 1, 219, (2000).

[17] S.L. Yuan, C.S. Xiong, Z.Y. Li, Z.C. Xia, G.Q. Zhang, G. Peng, F. Tu, Y.P. Yang, J. Liu, L. Liu, Y.H. Xiong. J. Phys.: Condens. Matter. 14, 173 (2002).

[18] Р.В. Демин, Л.И. Королева, А.З. Муминов, Я.М. Муковский. ФТТ 48, 305 (2006).

[19] H. Tanaka, T. Kanki, J.-H. Choi, T. Kawai, J. Zhang. Phys. Rev. B 64, 184404 (2001).

[20] Г.А. Овсянников, Т.А. Шайхулов, В.А. Шахунов, В.В. Демидов, Н.В. Андреев, А.Е. Пестун, В.Л. Преображенский. ФTT 59, 2178 (2017).

[21] G.A. Ovsyannikov, T.A. Shaikhulov, V.A. Shakhunov, V.L. Preobrazhensky T. Mathurin, N. Tiercelin, P. Pernod. J. Superconduct. Nov. Magn. 14, 173 (2019).

[22] Feifei Wang, Laihui Luo, Dan Zhou, Feifei Wang, Laihui Luo, Dan Zhou. Appl. Phys. Lett. 90, 212903 (2007).

[23] J. Zhang, H. Tanaka, T. Kanki, J.-H. Choi, T. Kawai. Phys. Rev. B 64. 184404 (2001).

[24] N.G. Bebenin, R.I. Zainullina, V.V. Mashkautsan, V.V. Ustinov, Ya.M. Mukovskii. Phys. Rev. B 69, 104434 (2004).

[25] T.X. Nan, Z.Y. Zhou, J. Lou, M. Liu, X. Yang, Y. Gao, S. Rand, N.X. Sun. Appl. Phys. Lett. 100, 132409 (2012).
[26] T. Wu, P. Zhao, M. Bao, A. Bur, J.L. Hockel, K. Wong, K.P. Mohanchandra, C.S. Lynch, G.P. Carman. J. Appl. Phys. 109, 124101 (2011).

[27] Weiping Zhou, Yuanqiang Xiong, Zhengming Zhang, Dunhui Wang, Weishi Tan, Qingqi Cao, Zhenghong Qian, Youwei Du. ACS Appl. Mater. Interface, 8, 5424 (2016).

[28] R.K. Zheng, Y. Jiang, Y. Wang, H.L.W. Chan. C.L. Choy, H.S. Luo. Phys. Rev. B 79, 174420 (2009).

[29] Н.Г. Бебенин, Р.И. Зайнуллина, В.В. Устинов. УФН 188, 801 (2018).

Редактор К.В. Емцев 